\title{
An Efficient Feedback Method for MIMO Systems with Slowly Time-Varying Channels
}

\author{
June Chul Roh and Bhaskar D. Rao \\ Department of Electrical and Computer Engineering \\ University of California, San Diego \\ La Jolla, CA 92093-0407, USA \\ Email: jroh@ece.ucsd.edu, brao@ece.ucsd.edu
}

\begin{abstract}
The capacity of a multiple-input multiple-output (MIMO) channel can be improved if the transmitter has knowledge of channel. In this paper, we propose an efficient and practical feedback method based on parameterization and quantization of channel parameters. The spatial information of channel at transmitter, which is represented as a matrix with orthonormal columns, has a geometrical structure. In parameterization, the geometrical structure is exploited to extract a set of parameters that has a one-to-one mapping to the original matrix. In slowly time-varying channels, the parameters are also found to be smoothly changing in time. We employ adaptive Delta modulation to quantize and feed back each parameter. The results show that the proposed feedback scheme has a channel tracking feature and achieves a capacity very close to the perfect feedback case with a reasonable feedback rate.
\end{abstract}

\section{INTRODUCTION}

Multiple transmit and receive antenna system is considered as a strong candidate for future wireless systems because of potential improvement in channel capacity and link performance. A multiple antenna channel provides different capacities under different channel state information (CSI) assumptions. The two common CSI assumptions are i) complete CSIT (channel state information at the transmitter) where perfect channel information is known to both the transmitter and the receiver, e.g., [1], [2]; and ii) no CSIT where perfect channel information is available only at the receiver, e.g., [1]. The former case, of course, provides a higher channel capacity than the latter, but the gain comes at an expense of the transmitter's perfect knowledge of MIMO channel. However, since in many applications the channel information is provided to the transmitter through a dedicated feedback channel, it is almost impossible for the transmitter to have perfect information in time-varying channels. Many previous studies considered the above two extreme CSIT assumptions, and there are only a few studies dealing with how to feed back the MIMO channel information. Some researchers have worked on feedback of channel information in vector form, for example, for multipleinput single-output (MISO) channels [3] and for the principal eigen-mode of MIMO channels [4]. Onggosanusi and Dabak [5] studied feedback of matrix channel information for MIMO channels. They introduced a feedback scheme where among a set of unitary matrices for the channel spatial information, an index of the matrix minimizing error probability is fed back to the transmitter.
The purpose of this paper is to provide a general framework for quantization of MIMO channel information and to develop a practical feedback method for slowly time-varying channels. The CSIT consists of the spatial information of channel and the power allocation over spatial channels. We first focus on quantization of the spatial information which can be represented as a matrix with orthonormal columns (a unitary matrix is an example). We notice a geometrical structure in the matrix. For example, the columns of a $t \times t$ unitary matrix $V=\left[v_{1}, \ldots, v_{t}\right]$ are all on the unit-norm sphere $\mathcal{S}_{t} \subset \mathbb{C}^{t}$ and mutually orthogonal, i.e., $v_{1} \in \mathcal{S}_{t}, v_{2} \in\left(\mathcal{S}_{t} \cap v_{1}^{\perp}\right)$, $v_{3} \in\left(\mathcal{S}_{t} \cap v_{1}^{\perp} \cap v_{2}^{\perp}\right)$, and so on, where $v_{i}^{\perp}$ is the orthogonal complement of the space spanned by $v_{i}$. In this paper, the geometrical structure is exploited in quantizing the spatial information. In particular, from the matrix with orthonormal columns, we extract a set of essential parameters that has a one-to-one mapping to the original matrix. The number of parameters equals the degree of freedom in the matrix. Then, instead of quantizing the original matrix, the parameters are quantized and fed back to the transmitter, and an approximate (quantized) version of spatial information is reconstructed at the transmitter. Although jointly quantizing the parameters (vector quantization) could be better choice, this paper considers quantizing each parameter independently (scalar coding) because of its low complexity. More specifically, adaptive Delta modulation (ADM) [6] is employed from an observation that, in slowly time-varying channels, the extracted parameters are also smoothly changing. ADM is a practical low-rate scalar coding scheme that can track time-varying channels efficiently.

We use the following notations. $A^{\dagger}$ and $A^{T}$ indicate the conjugate transpose and the transpose of matrix $A$, respectively. $I_{n}$ is the $n \times n$ identity matrix and $0_{m, n}$ means the $m \times n$ zero matrix. $\operatorname{diag}\left(a_{1}, \ldots, a_{n}\right)$ is a square diagonal matrix with $a_{1}, \ldots, a_{n}$ along the diagonal. The 2-norm of vector $v$ is denoted by $\|v\| . E[\cdot]$ represents the expectation operator, and $\mathcal{C N}(\mu, \Sigma)$ is circularly symmetric complex Gaussian random vector with mean $\mu$ and covariance $\Sigma$.

\section{System Model And Mutual Information}

\section{A. Channel Model}

We consider a multiple antenna system with $t$ antennas at the transmitter and $r$ at the receiver. Assuming slow flat-fading, the MIMO channel is modeled by the channel matrix $H \in$ 
$\mathbb{C}^{r \times t}$. That is, the channel input $x \in \mathbb{C}^{t}$ and the channel output $y \in \mathbb{C}^{r}$ have the following relationship:

$$
y=H x+\eta
$$

where $\eta \in \mathbb{C}^{r}$ is the additive white Gaussian noise vector distributed by $\mathcal{C N}\left(0_{r, 1}, I_{r}\right)$. We denote the rank of $H$ by $m$. And, the singular value decomposition (SVD) of $H$ is given by $H=U_{H} \Sigma_{H} V_{H}^{\dagger}$, where $U_{H} \in \mathbb{C}^{r \times r}$ and $V_{H} \in \mathbb{C}^{t \times t}$ are unitary matrices and $\Sigma_{H} \in \mathbb{R}^{r \times t}$ contains the singular values $\sigma_{1} \geq \ldots \geq \sigma_{m}>0$ of $H$. We impose a constraint on the transmit power by $E\left[x^{\dagger} x\right] \leq P_{T}$.

We assume that in all cases perfect CSI is known to the receiver. And, the first $n(0 \leq n \leq m)$ columns of $V_{H}$ are to be quantized and fed back to the transmitter as channel spatial information. When we consider perfect feedback, i.e., no quantization error, this setting includes the two extreme cases: i) $n=m$ is the case that the transmitter has same spatial information as in the complete CSIT case; and ii) $n=0$ accounts for no spatial information at the transmitter as in the no CSIT case. And, when $0<n<m$, it corresponds to partial CSIT of [7], [8]. For notational convenience, let us define $V=\left[v_{1}, \ldots, v_{n}\right]$ where $v_{i}$ is the $i$-th column vector of $V_{H}$.

The CSIT consists of the spatial information of channel and the power allocation information. The matrix $V$ conveys the spatial information that is needed at the transmitter. In [7], we discussed a multiple-antenna system concept in which the optimal power allocation is calculated at the receiver and provided to the transmitter as additional CSI. The power allocation information is represented by a real vector $\gamma=\left[\gamma_{i}\right]$ where $\sum_{i} \gamma_{i}=1$ and $0 \leq \gamma_{i} \leq 1$.

\section{B. Feedback System Model}

This subsection describes a feedback system model for timevarying MIMO channels that accounts for the discrepancy between the real channel and the CSI at the transmitter. It will be used in performance evaluation in Section V. Figure 1 depicts the block-fading model and the frame structure of the feedback system model. We assume that the channel matrix is not changing during a time block, which will be called channel block (with length $T_{C}$ ). The channel matrix at $k$ th channel block is denoted by $H[k]$, and $V[k]$ and $\gamma[k]$ are the corresponding CSI. The quantized version of the CSI $(\hat{V}[k]$ and $\hat{\gamma}[k]$ in the figure) is provided to the transmitter at a feedback rate of $R_{F}$ times per second via an error-free feedback channel. The time frame between two consequent channel updates is called feedback frame (with length $T_{F}=$ $\left.1 / R_{F}\right)$. For simplicity, we assume there are $M$ (an integer) channel blocks in a feedback frame, i.e., $T_{F}=M T_{C}$. In addition, in order to model composite delay, e.g., due to processing and propagation, we introduce an integer parameter $D$ : at the starting point of each frame, the CSI corresponding to the $D$ previous channel block is available at the transmitter. Figure 1 is an example when $D=1$. The CSIT is used in transmission during the frame before the next update arrives.

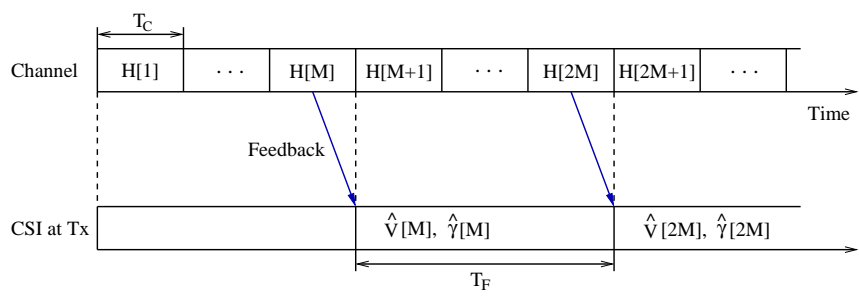

Fig. 1. Feedback system model (when $D=1$ ).

\section{Mutual Information and Capacity}

Among many possible measures for evaluating the performance of the feedback method, we consider mutual information as performance measure in this paper. When the transmit signal $x$ is distributed by $\mathcal{C N}\left(0_{t, 1}, \Phi_{x}\right)$, the mutual information for a given channel realization $H$ is given by $I(x ; y)=$ $\log \operatorname{det}\left(I_{r}+H \Phi_{x} H^{\dagger}\right)$ [1]. Since the covariance matrix $\Phi_{x}$ is a Hermitian positive semidefinite matrix, it can be decomposed as $\Phi_{x}=W \Phi W^{\dagger}$ with a unitary matrix $W \in \mathbb{C}^{t \times t}$ and a diagonal matrix $\Phi=\operatorname{diag}\left(P_{1}, \ldots, P_{t}\right), P_{i} \geq 0$. From this, we notice that it is equivalent to transmitting $x=W s, s \in \mathbb{C}^{t}$ with $E\left[s s^{\dagger}\right]=\Phi$ over channel $H$, i.e., an equivalent channel is

$$
y=H W s+\eta .
$$

This point of view is useful because each column of $W$ can be interpreted as the beamforming vector for the corresponding symbol in $s$. And, in some cases, $W$ and $\Phi$ can be adjusted by using the spatial and the power allocation information available at the transmitter. Let us define $\gamma=\left[\gamma_{1}, \ldots, \gamma_{t}\right]$, where $\gamma_{i} \in[0,1]$ and $\sum_{i} \gamma_{i}=1$, which is referred as power allocation information by setting $\gamma_{i}=P_{i} / P_{T}$, i.e., $\Phi(\gamma)=P_{T} \operatorname{diag}\left(\gamma_{1}, \ldots, \gamma_{t}\right)$. And, we denote the mutual information $I(s ; y)$ of the channel (2) when the transmitter uses beamforming matrix $W$ and power allocation $\gamma$ by

$$
\begin{aligned}
\Psi(W, \gamma) & =\log \operatorname{det}\left(I+H W \Phi(\gamma) W^{\dagger} H^{\dagger}\right) \\
& =\log \operatorname{det}\left(I+V_{H}^{\dagger} W \Phi(\gamma) W^{\dagger} V_{H} \Sigma^{2}\right) .
\end{aligned}
$$

When the transmitter has perfect knowledge of channel (as in complete CSIT case), $W$ is set to $V_{H}$. Then, the mutual information is written as $I(s ; y)=\Psi\left(V_{H}, \gamma\right)=\sum_{i=1}^{m} \log (1+$ $\left.P_{i} \lambda_{i}\right)$, where $\lambda_{i}=\sigma_{i}^{2}$. With water-filling to maximize $I(s ; y)$, we have the channel capacity

$$
C_{\text {Full }}=\sum_{i=1}^{m}\left[\log \left(\nu \lambda_{i}\right)\right]^{+}
$$

where $[a]^{+}$is defined as $\max \{a, 0\}$ and $\nu$ is the water-filling level satisfying the power constraint $\sum_{i=1}^{m}\left[\nu-\lambda_{i}^{-1}\right]^{+}=P_{T}$. When we denote the optimum power allocation information by $\gamma_{\mathrm{wf}}$, we can write $C_{\text {Full }}=\Psi\left(V_{H}, \gamma_{\mathrm{wf}}\right)$. On the other hand, when no information about channel is available at the transmitter (as in no CSIT case), the capacity is given by

$$
C_{\text {None }}=\Psi\left(I_{t}, \gamma_{\text {unif }}\right)=\sum_{i=1}^{m} \log \left(1+\frac{P_{T}}{t} \lambda_{i}\right)
$$


where $\gamma_{\text {unif }}=[1 / t, \ldots, 1 / t]$. These two capacities for the extreme cases will be used as references in comparing performances.

Now, we consider the following two scenarios where some non-perfect channel information is available at the transmitter. The first case is when the transmitter uses the quantized and/or delayed version of spatial information $\hat{V}_{H}$ and power allocation information $\hat{\gamma}$. Then, the mutual information can be written as

$$
I_{\hat{V}_{H}, \hat{\gamma}}=\Psi\left(\hat{V}_{H}, \hat{\gamma}\right) .
$$

Note that the subscripts in mutual information and capacity notations indicate the CSIT. The second case is when the transmitter has only spatial information $\hat{V}_{H}$ and no power allocation information. In this case, one easy choice of power allocation is uniform allocation. Then, the mutual information is given by

$$
I_{\hat{V}_{H}}=\Psi\left(\hat{V}_{H}, \gamma_{\text {unif }}\right)=\sum_{i=1}^{m} \log \left(1+\frac{P_{T}}{t} \lambda_{i}\left(H_{\text {eq }} H_{\text {eq }}^{\dagger}\right)\right)
$$

where $H_{\mathrm{eq}}=H \hat{V}_{H}$ and $\lambda_{i}\left(H_{\mathrm{eq}} H_{\mathrm{eq}}^{\dagger}\right)$ is the $i$-th largest eigenvalue of $H_{\mathrm{eq}} H_{\mathrm{eq}}^{\dagger}$.

We expect that channel feedback has more gain when $t>r$ [7]. In this case, since the rank of channel $m<t$, we need to feedback only first $m$ columns of $V_{H}$, i.e., $V=\left[v_{1}, \ldots, v_{m}\right]$ $(n=m)$. And, if $\hat{V}$ is reasonably close to $V$, the optimum power allocation will has nearly zeros in the last $t-m$ entries in $\gamma$. Therefore, when only spatial information $\hat{V}$ is available at the transmitter, a reasonable uniform power allocation is $\gamma=[1 / m, \ldots, 1 / m, 0, \ldots, 0]$.

\section{Parameterization of Channel Information}

In this section, we focus on how to extract essential parameters from the spatial information denoted by $V$. Since the columns in spatial information $V$ are geometrically structured, the degree of freedom in the matrix is much smaller than the number of real-number entries in the matrix. The degree of freedom in $V \in \mathbb{C}^{t \times n}$ can be expressed as

$$
N=2 t \cdot n-n-2\left(\begin{array}{l}
n \\
2
\end{array}\right)=2 t n-n^{2} \text { (real numbers) }
$$

where the first term is the number of real-number entries in the matrix, and second term accounts for reductions from unit-norm property of each column, and third term from orthogonality in each pair of columns. For example, a $t \times t$ unitary matrix has $2 t^{2}$ real-number entries, but its degree of freedom is only $t^{2}$. Furthermore, one phase in each column can be made fixed (e.g., the first row has all nonnegative real numbers), which gives $n$ additional reductions. Then,

$$
N=(2 t-1) n-n^{2} \text { (real numbers) }
$$

Now, we want to extract a set of essential parameters that has a one-to-one mapping to the matrix $V$. There are several possible ways such as using Givens rotations or Householder reflections. In this paper, we propose a parameterization method using Givens rotations in which the number of parameters is equal to (8), the degree of freedom in the matrix.

Theorem 1 (Parameterization): A matrix $V \in \mathbb{C}^{t \times n}(t \geq$ $n$ ) with orthonormal columns can be decomposed as

$$
V=\left[\prod_{k=1}^{n} D_{k}\left(\phi_{k, k}, \ldots, \phi_{k, t}\right) \prod_{l=1}^{t-k} G_{t-l, t-l+1}\left(\theta_{k, l}\right)\right] \tilde{I}
$$

where $t$ dimensional diagonal matrix

$$
D_{k}\left(\phi_{k, k}, \ldots, \phi_{k, t}\right)=\operatorname{diag}\left(1_{k-1}, e^{j \phi_{k, k}}, \ldots, e^{j \phi_{k, t}}\right)
$$

$1_{k-1}$ is $(k-1) 1$ 's; and $G_{p-1, p}(\theta)$ is the Givens matrix which operates in the $(p-1, p)$ coordinate plane of the form

$$
G_{p-1, p}(\theta)=\left[\begin{array}{cccc}
I_{p-2} & & & \\
& c & -s & \\
& s & c & \\
& & & I_{t-p}
\end{array}\right],
$$

$c=\cos \theta$ and $s=\sin \theta$; and $t \times n$ matrix $\tilde{I}=\left[I_{n}, 0_{n, t-n}\right]^{T}$.

Let us explain the above parameterization procedure with an example. Consider $4 \times 3$ matrix $V$ with orthonormal columns.

$$
\begin{aligned}
& {\left[\begin{array}{ccc}
\times & \times & \times \\
\times & \times & \times \\
\times & \times & \times \\
\times & \times & \times
\end{array}\right] \stackrel{D_{1}^{\dagger}}{\longrightarrow}\left[\begin{array}{c|cc}
|\times| & \times & \times \\
\times & \times & \times \\
\times & \times & \times \\
\times & \times & \times
\end{array}\right] \stackrel{G_{3,4}^{\dagger}, G_{2,3}^{\dagger}, G_{1,2}^{\dagger}}{\longrightarrow}\left[\begin{array}{ccc}
1 & 0 & 0 \\
0 & \times & \times \\
0 & \times & \times \\
0 & \times & \times
\end{array}\right] \stackrel{D_{2}^{\dagger}}{\longrightarrow}} \\
& {\left[\begin{array}{ccc}
1 & 0 & 0 \\
0 & |\times| & \times \\
0 & |\times| & \times \\
0 & |\times| & \times
\end{array}\right] \stackrel{G_{3,4}^{\dagger}, G_{2,3}^{\dagger}}{\longrightarrow}\left[\begin{array}{ccc}
1 & 0 & 0 \\
0 & 1 & 0 \\
0 & 0 & \times \\
0 & 0 & \times
\end{array}\right] \stackrel{D_{3}^{\dagger}}{\longrightarrow}\left[\begin{array}{ccc}
1 & 0 & 0 \\
0 & 1 & 0 \\
0 & 0 & |\times| \\
0 & 0 & |\times|
\end{array}\right] \stackrel{G_{3,4}^{\dagger}}{\longrightarrow} \tilde{I}}
\end{aligned}
$$

where $|\times|$ represents the magnitude of a particular element. The procedure is similar to the $\mathrm{QR}$ decomposition using Givens matrices. In the first step, we want to make all the entries in the first column under the first component all zeros. To do that, we first extract the phases from the first column by pre-multiplying $V$ by $D_{1}^{\dagger}$ to have a real-valued column, and then apply a series of Givens matrices with appropriate parameters to make all entries under the $(1,1)$ element zeros. Since the Givens rotation preserves the length of vector, the $(1,1)$ element becomes 1 . At the same time, all the entries in the first row except the $(1,1)$ element also become zeros because of the orthogonality between columns. We carry out similar procedures on the remaining columns sequentially, and then finally we have a diagonal matrix $\tilde{I}$. Since a Givens matrix is an orthogonal matrix, the matrix $V$ can be factored as

$$
\begin{aligned}
V= & D_{1}\left(\phi_{1,1}, \ldots, \phi_{1,4}\right) G_{3,4}\left(\theta_{1,1}\right) G_{2,3}\left(\theta_{1,2}\right) G_{1,2}\left(\theta_{1,3}\right) \\
& \cdot D_{2}\left(\phi_{2,2}, \phi_{2,3}, \phi_{2,4}\right) G_{3,4}\left(\theta_{2,1}\right) G_{2,3}\left(\theta_{2,2}\right) \\
& \cdot D_{3}\left(\phi_{3,3}, \phi_{3,4}\right) G_{3,4}\left(\theta_{3,1}\right) \tilde{I} .
\end{aligned}
$$

Therefore, once we have a set of parameters, the phases $\left\{\phi_{k, l}\right\}$ and the rotation angles $\left\{\theta_{k, l}\right\}$, the original matrix $V$ can be exactly reconstructed.

Now, we will show that the number of parameters obtained by the proposed parameterization is equal to the degree of freedom in $V$ with the following with the following Lemma and Theorem.

Lemma 1: Define as $\tilde{V}$ the resulting matrix after applying $D_{1}$ and the first $l$ Givens rotations $G_{t-q, t-q+1}^{\dagger}\left(\theta_{1, q}\right), q=$ 
$1, \ldots, l$, in the procedure for the first column. Then, the $(t-$ $l, p)$ element of $\tilde{V}$ is given by

$$
\tilde{V}[t-l, p]= \begin{cases}\left\|v_{1}^{(l+1)}\right\| & \text { if } p=1, \\ \frac{\left(v_{1}^{(l+1)}\right)^{\dagger} v_{p}^{(l+1)}}{\left\|v_{1}^{(l+1)}\right\|} & \text { if } p=2, \ldots, n\end{cases}
$$

where $v_{i}^{(l+1)}$ is a vector defined as the last $l+1$ elements in the $i$-th column $v_{i}$, i.e., $v_{i}^{(l+1)}=\left[v_{t-l, i}, v_{t-l+1, i}, \ldots, v_{t, i}\right]^{T}$.

Proof: This can be proved by induction.

Theorem 2: In the matrix factorization of Theorem 1, if orthonormal column matrix $V$ has real-valued elements in the first row with alternating signs as $+-+-\ldots$, then the first parameter of $D_{k}$ is zero, i.e., $\phi_{k, k}=0$ for all $k$. Therefore, the number of parameters is $(2 t-1) n-n^{2}$, which is the degree of freedom in $V$.

Proof: This can be proved by using Lemma 1 and orthogonality between two columns of $V$. After applying $D_{1}^{\dagger}$ and $t-1$ Givens matrices $G_{t-l, t-l+1}^{\dagger}\left(\theta_{1, l}\right), l=1, \ldots, t-1$, it can be shown that the resulting matrix is given by

$$
G_{1,2}^{\dagger} \ldots G_{t-1, t}^{\dagger} D_{1}^{\dagger} V=\left[\begin{array}{cc}
1 & 0_{1, n-1} \\
0_{t-1,1} & V^{\prime}
\end{array}\right],
$$

and $V^{\prime}$ is a $(t-1) \times(n-1)$ matrix with orthonormal columns that has same structure as $V$, i.e., alternating signs in the first row. Therefore, in a sequential way we can prove the first phase parameter of $D_{k}$ is zero for all $k$. From this, we have $n$ less parameters than (7), which results in the final conclusion.

Now we find the distribution of the parameters and establish their independence, a property useful for quantization purposes.

Theorem 3 (Statistics of Parameters): When the channel matrix $H$ has i.i.d. $\mathcal{C N}(0,1)$ entries, then all the parameters from Theorem 1 are statistically independent. Moreover, the phase $\phi_{k, j}$ is uniformly distributed over $(-\pi, \pi]$ for all $k$ and $j$, and the rotational angle $\theta_{k, l}$ has probability density

$$
p\left(\theta_{k, l}\right)=2 l \sin ^{2 l-1} \theta_{k, l} \cos \theta_{k, l}, \quad 0 \leq \theta_{k, l}<\frac{\pi}{2} .
$$

Proof: The theorem can be proved using techniques for calculating the distribution of transformed random vector/matrix similar to [9, Ch. 1-3]. Details are omitted due to space limitations.

The parameterization for power allocation information $\gamma=$ $\left[\gamma_{1}, \ldots, \gamma_{t}\right], \sum_{i=1}^{t} \gamma_{i}=1$ is rather simple. We can see that $\gamma$ has $t-1$ of degree of freedom. And, the parameters can be simply the first $t-1$ elements, $\left[\gamma_{1}, \ldots, \gamma_{t-1}\right]$. Then, from the constraint, the last one is determined as $\gamma_{t}=1-\sum_{i=1}^{t-1} \gamma_{i}$.

\section{Quantization in Parameter Domain}

The overall strategy for quantization is depicted in Figure 2 and summarized below.

1) From the spatial information $V$, extract a set of parameters $\Theta$ (Parameterization): $\Theta=\mathcal{T}(V)$.

2) Quantize the parameters $\Theta$ and feed back the quantized parameters $\hat{\Theta}$ (Quantization): $\hat{\Theta}=\mathcal{Q}(\Theta)$.
3) Reconstruct the spatial information $\hat{V}$ from $\hat{\Theta}$ (Reconstruction): $\hat{V}=\mathcal{T}^{-1}(\hat{\Theta})$.

The proposed methodology for quantization has many advantages. Some of them are as follows. The number of parameters to quantize is minimal since it equals the degree of freedom in the spatial information. The parameters, which are phases and angles, are all bounded quantities. The reconstructed matrix $\hat{V}$ has the same geometrical structure as $V$, i.e., $\hat{V}^{\dagger} \hat{V}=I_{n}$. In addition, the methodology is general and can be applied to any multiple antenna scenario: MISO systems (when $n=1$ ) and MIMO systems with partial feedback (when $1<n<m$ ) as well as MIMO systems with full feedback $(n=m)$.

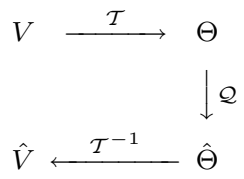

Fig. 2. Quantization in the parameter domain.

One can apply some vector quantization (VQ) method to quantize the parameters. But, complexity issues and tracking requirement motivate us to consider employing scalar quantization. Moreover, the independence of the parameters, Theorem 3, indicates that the overall loss is minimal. More specifically, adaptive Delta modulation (ADM) [6, ch. 8] is used to quantize each parameter. In slowly time-varying channels, the parameters are also slowly and continuously changing most of the time. The encoder of ADM consists of a simple accumulator and a one-bit quantizer. Basically, it quantizes the difference between the newly incoming sample and the previous quantized sample. For a parameter $\theta$,

$$
\hat{\theta}[k]=\hat{\theta}[k-1] \pm \Delta[k] .
$$

And the step-size $\Delta[k]$ of the one-bit quantizer is adaptively changing to better track the dynamics of the signal. The ADM with one-bit memory [6] is an example. The step-size is increased if the consequent two encoded bits are same, and decreased otherwise, that is,

$$
\Delta[k]= \begin{cases}M_{1} \Delta[k-1], & \text { if } c[k]=c[k-1] \\ M_{2} \Delta[k-1], & \text { if } c[k] \neq c[k-1]\end{cases}
$$

where $\Delta[k]$ and $c[k]$ is the step-size and the encoded bit for the $k$-th sample; and $M_{1}>1$ and $0<M_{2}<1$, usually $M_{2}=1 / M_{1}$. Compared to VQ, ADM has considerably lower complexity. And it is a low-rate scalar quantization scheme (as low as one bit per parameter). Another important advantage is that ADM has inherently a channel tracking feature for slowly time-varying channels.

\section{Numerical Results}

We have performed simulations to investigate the performance of the proposed feedback method, especially in slowly time-varying MIMO channels. The components of the channel matrix are i.i.d. discrete-time random processes and each process models Rayleigh fading channel gain. The simulated 
channel has the Doppler frequency $f_{D}=7.4 \mathrm{~Hz}$, which corresponds to a mobility of $4 \mathrm{~km} / \mathrm{h}$ at carrier frequency of 2 $\mathrm{GHz}$. As for the frame structure of Section II-B, we considered the case of $M=4$ and $D=1$.

Figure 3 shows the cumulative distribution of mutual information with different CSIT assumptions and various transmit power, $P_{T}=-10,0,10,20 \mathrm{~dB}$, with the feedback rate $R_{F}=$ 500 per second $(t=4$ and $r=2)$. $C_{\text {Full }}$ and $C_{\text {None }}$ are calculated from (3) and (4), respectively. Note that the CSIT for $C_{\text {Full }}$ is perfect, that is, it involves neither quantization error nor channel tracking error. The performances of the proposed feedback method are shown as $C_{\hat{V}, \hat{\gamma}}$ and $C_{\hat{V}}$, which are calculated according to (5) and (6), respectively. These include the effect of quantization error and delay; therefore, they reflect more practical situations of feedback systems. From the results, we can see that, in low transmit power range, the two have some gap; but, in high transmit power range, the two have little difference. This means that power allocation information is important in low transmit power range, which can be understood from the water-filling argument. That is, when transmit power is low, the optimum transmission scheme is using only a few spatial channels that have high channel gains. Note that the feedback rate is corresponding to $5.5 \mathrm{kbps}$ (for $C_{\hat{V}, \hat{\gamma}}$ ) and $5 \mathrm{kbps}$ (for $C_{\hat{V}}$ ) of feedback bit-rate since we have 10 parameters for $V$ and one for $\gamma$, and ADM encodes each parameter into one bit at each feedback instant.

Figure 4 shows the results when the feedback rate is increased to $R_{F}=1000$ per second, which corresponds to $11 / 10$ kbps. We can see that the performances become much closer to $C_{\text {Full }}$. This can be explained as follows. By increasing the feedback rate, the quantization error is reduced, since in ADM encoding the variations between the adjacent samples are reduced. Also the channel tracking error due to delay is lessened with increasing the feedback rate.

\section{CONCLUSION}

We proposed a general framework for quantization of MIMO channel information, which involves parameterization of orthonormal column matrix and quantization of parameters. We introduced a new parameterization method that uses Givens rotations and that provides minimal number of parameters. The distributions of the parameters were found and the independence between them was shown. In slowly timevarying channels, the extracted parameters are also slowly and continuously changing in time. This motivated employing adaptive Delta modulation in quantizing the parameters. The adaptive Delta modulation is a simple and practical quantization method that has a channel tracking feature for slowly time-varying channels. The proposed feedback scheme requires $(2 t-1) n-n^{2}$ bits to feedback $V \in \mathbb{C}^{t \times n}$. With the proposed feedback method, a performance close to the perfect feedback case can be achieved with a reasonable feedback rate.

\section{ACKNOWLEDGMENT}

This research was supported by CoRe grant No. 02-10109 sponsored by Ericsson.

\section{REFERENCES}

[1] I. E. Telatar, "Capacity of multi-antenna Gaussian channels," AT\&T Bell Labs Tech. Memo., 1995.

[2] E. Biglieri, G. Caire, and G. Taricco, "Limiting performance of blockfading channels with multiple antennas," IEEE Trans. Inform. Theory, vol. 47, no. 4, pp. 1273-1289, May 2001.

[3] K. K. Mukkavilli, A. Sabharwal, E. Erkip, and B. Aazhang, "On beamforming with finite rate feedback in multiple antenna systems," IEEE Trans. Inform. Theory, vol. 49, no. 10, pp. 2562 - 2579, Oct. 2003.

[4] D. J. Love, R. Heath, Jr., and T. Strohmer, "Grassmannian beamforming for multiple-input multiple-output wireless systems," IEEE Trans. Inform. Theory, vol. 49, no. 10, pp. 2735 - 2747, Oct. 2003.

[5] E. N. Onggosanusi and A. G. Dabak, "A feedback-based adaptive multiinput multi-output signaling scheme," in Proc. Asilomar Conf. 2002, Pacific Grove, CA, Nov. 2002.

[6] N. S. Jayant and P. Noll, Digital Coding for Waveforms: Principles and Applications to Speech and Video. Prentice-Hall, 1984.

[7] J. C. Roh and B. D. Rao, "Multiple antenna channels with partial channel state information at the transmitter," IEEE Trans. Wireless Commun., Mar. 2004.

[8] C. Murthy, J. C. Roh, and B. D. Rao, "Optimality of extended maximum ratio transmission," in 6th Baiona Workshop on Signal Processing in Communications, Baiona, Spain, Sept. 2003.

[9] R. J. Muirhead, Aspects of Multivariate Statsitical Theory. John Wiley \& Sons, 1982.

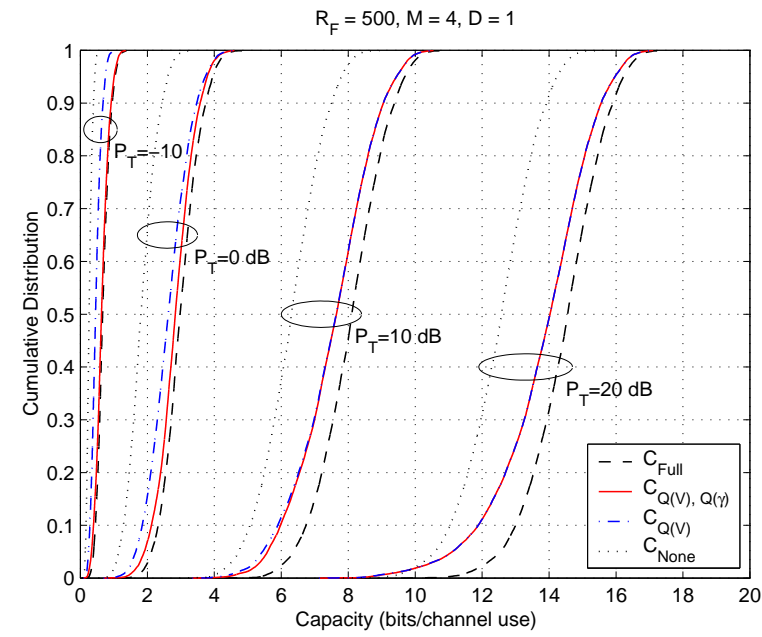

Fig. 3. Cumulative distribution of capacities when $R_{F}=500 / \mathrm{sec}(t=4$ and $r=2) . Q(V)=\hat{V}$ and $Q(\gamma)=\hat{\gamma}$.

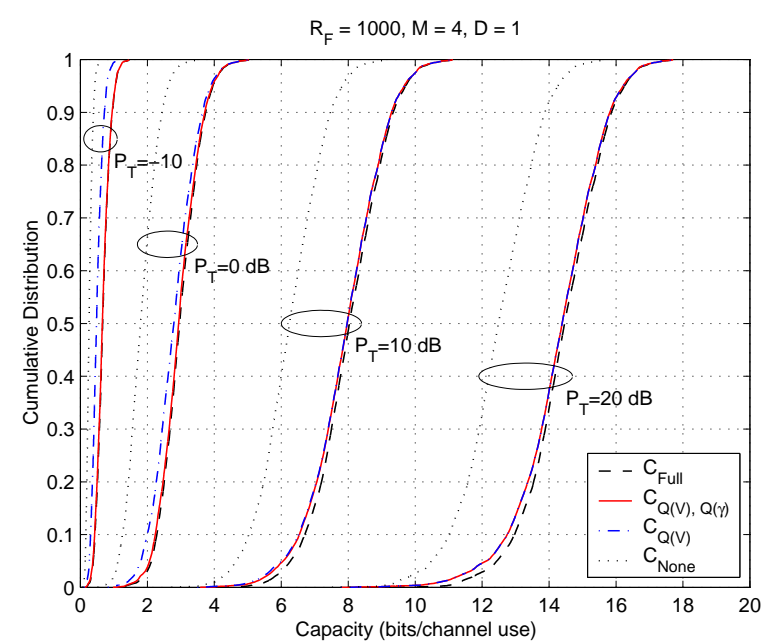

Fig. 4. Cumulative distribution of capacities when $R_{F}=1000 / \mathrm{sec}(t=4$ and $r=2$ ). 